\title{
Ideal Method for Small Bowel Preparation before Video Capsule Endoscopy
}

\author{
Jun Lee ${ }^{1}$ and Shai Friedland ${ }^{2,3}$ \\ ${ }^{1}$ Department of Internal Medicine, Chosun University, Gwangju, Korea, ${ }^{2}$ Department of Medicine, Stanford University, Palo Alto, CA, \\ USA, ${ }^{3}$ Gastroenterology Section, VA Palo Alto Health Care System, Palo Alto, CA, USA
}

See “The Efficacy of 4 Liters of Clear Liquids for Small Bowel Preparation Prior to Video Capsule Endoscopy" by Nicholas Placone, Runalia Bahar, and Surinder Mann, on page 713-718.

Video capsule endoscopy is a novel, noninvasive method for directly visualizing the small intestine. It is widely used for various indications including obscure gastrointestinal bleeding, iron deficiency anemia, Crohn's disease, and evaluation of small intestinal tumors. ${ }^{1}$ To increase the diagnostic yield of video capsule endoscopy, it is essential to remove obstacles to mucosal visualization such as food ingredients, bile, and air bubbles. Several systematic reviews and meta-analyses have shown that purgative bowel preparation improves mucosal visualization compared with fasting or clear liquid diets. ${ }^{2,3}$ Based on these results, multiple guidelines such as those of the Korean Society of Gastrointestinal Endoscopy (KSGE), American Society of Gastroenterology (ASGE), and European Society of Gastrointestinal Endoscopy (ESGE) recommend purgative bowel preparation prior to video capsule endoscopy for better mucosal visualization. ${ }^{1,4,5}$ However, the meta-analyses are not highly reliable as they included studies with heterogeneous protocols, small sample sizes, and different endpoints. There is insufficient evidence to claim that purgative bowel preparation

\footnotetext{
Received: October 2, 2020 Revised: October 9, 2020

Accepted: October 9, 2020

Correspondence: Shai Friedland

Gastroenterology Section, VA Palo Alto Health Care System, GI-111, Palo Alto, CA 94304, USA

Tel: +1-650-725-8117, Fax: +1-650-321-7917, E-mail: Shai_friedland@yahoo. com

ORCID: https://orcid.org/0000-0002-9782-000X
}

(c) This is an Open Access article distributed under the terms of the Creative Commons Attribution Non-Commercial License (http://creativecommons.org/ licenses/by-nc/3.0) which permits unrestricted non-commercial use, distribution, and reproduction in any medium, provided the original work is properly cited. improves the diagnostic yield and completion rate of video capsule endoscopy. In addition, other studies have reported that purgative bowel preparation may not be superior to clear liquid diets. ${ }^{6,7}$

In this issue of Clinical Endoscopy, Placone et al. attempted to validate the efficacy of $4 \mathrm{~L}$ clear liquid preparation before video capsule endoscopy with a large sample size. ${ }^{8}$ Previously, the authors of this study conducted a randomized controlled study and reported that $4 \mathrm{~L}$ clear liquid preparation was not inferior to polyethylene glycol solution (PEG) in terms of image quality. ${ }^{9}$ In the current study, the 2 L PEG group and $4 \mathrm{~L}$ clear liquid group consisted of 284 consecutive patients who underwent video capsule endoscopy from June 2011 to December 2012 and from March 2017 to May 2018, respectively. Compared to the PEG group, the $4 \mathrm{~L}$ clear liquid group had a higher mean image quality score (2.98 vs. $2.67, p<0.0001)$ and adequate small bowel preparation ( $72 \%$ vs. $64 \%, p=0.0214)$. This study had significant limitations such as retrospective design, substantial difference in registration period and indications between the two groups, and low validation as a result of unblinded peer review of the manuscript by only one clinician. A particular concern is that the clear liquid diet group underwent capsule endoscopy in 2017-2018, while the purgative group underwent capsule endoscopy in 2011-2012, which could introduce significant biases due to the changes in patient referral patterns, upgrades in capsule endoscopy systems, improvements in image analysis software, and perhaps even the subconscious bias in rating bowel preparations. Nevertheless, this study has a relatively large sample size that can support the 
idea that $4 \mathrm{~L}$ of clear liquid could be effective for small bowel preparation.

In contrast to the previous meta-analysis, a recent meta-analysis of 12 eligible trials comparing purgative bowel preparation and clear liquid diet reported no statistically significant difference in diagnostic yield (risk ratio [RR], 1.17; 95\% confidence interval $[\mathrm{CI}], 0.97-1.40 ; p=0.11)$ and visualization quality (RR, $1.14 ; 95 \% \mathrm{CI}, 0.96-1.65){ }^{10}$ The authors attempted to minimize potential biases, and their results suggest that purgative preparations do not lead to superior diagnostic yield and mucosal visualization compared to clear liquid diet. ${ }^{10}$

To date, the ideal bowel preparation method before video capsule endoscopy remains controversial despite guidelines favoring purgative bowel preparation. In order to achieve consensus, it is necessary to establish a consistent protocol for small bowel preparation, including an appropriate diet and the optimal timing for taking purgatives. In addition, multicenter randomized controlled studies with adequate sample size, validated definition of diagnostic yield and visual quality, standardized purgative dose and regimen, and homogenous patient population are needed.

Conflicts of Interest

The authors have no financial conflicts of interest.

ORCID

Jun Lee: https://orcid.org/0000-0002-8060-9646

\section{REFERENCES}

1. Enns RA, Hookey L, Armstrong D, et al. Clinical practice guidelines for the use of video capsule endoscopy. Gastroenterology 2017;152:497-514.

2. Rokkas T, Papaxoinis K, Triantafyllou K, Pistiolas D, Ladas SD. Does purgative preparation influence the diagnostic yield of small bowel video capsule endoscopy?: A meta-analysis. Am J Gastroenterol 2009;104:219227.

3. Kotwal VS, Attar BM, Gupta S, Agarwal R. Should bowel preparation, antifoaming agents, or prokinetics be used before video capsule endoscopy? A systematic review and meta-analysis. Eur J Gastroenterol Hepatol 2014;26:137-145.

4. Song HJ, Moon JS, Do JH, et al. Guidelines for bowel preparation before video capsule endoscopy. Clin Endosc 2013;46:147-154.

5. Rondonotti E, Spada C, Adler S, et al. Small-bowel capsule endoscopy and device-assisted enteroscopy for diagnosis and treatment of small-bowel disorders: European Society of Gastrointestinal Endoscopy (ESGE) technical review. Endoscopy 2018;50:423-446.

6. Hookey L, Louw J, Wiepjes M, et al. Lack of benefit of active preparation compared with a clear fluid-only diet in small-bowel visualization for video capsule endoscopy: results of a randomized, blinded, controlled trial. Gastrointest Endosc 2017;85:187-193.

7. Pons Beltrán V, González Suárez B, González Asanza C, et al. Evaluation of different bowel preparations for small bowel capsule endoscopy: a prospective, randomized, controlled study. Dig Dis Sci 2011;56:29002905.

8. Placone N, Bahar R, Mann S. The efficacy of 4 liters of clear liquids for small bowel preparation prior to video capsule endoscopy. Clin Endosc 2020;53:713-718

9. Bahar R, Gupta A, Mann SK. Clear liquids versus polyethylene glycol preparation for video capsule endoscopy of the small bowel: a randomized controlled trial. Digestion 2019;99:213-218.

10. Gkolfakis P, Tziatzios G, Dimitriadis GD, Triantafyllou K. Meta-analysis of randomized controlled trials challenging the usefulness of purgative preparation before small-bowel video capsule endoscopy. Endoscopy 2018:50:671-683. 